\title{
Automated Crystal Orientation and Phase Mapping for Thin Film Applications by Transmission Electron Microscopy
}

\author{
E. F. Rauch *, K. Barmak**, J. K. Ganesh ***, P.J. Ferreira*** A. Darbal**, D. Choi**, T. \\ Sun****, B. Yao****, K. R. Coffey****, and S. Nicolopoulos***** \\ * SIMAP/GPM2 laboratory, Université de Grenoble/CNRS Grenoble INP - UJF, 38402 Saint- \\ Martin d'Hères, France \\ ** Carnegie Mellon University, Dept. of Materials Science and Eng., Pittsburgh, PA 15213 \\ *** Materials Science and Engineering, University of Texas at Austin, Austin, TX, 78712 \\ **** Advanced Materials and Processing Center, Univ. of Central Florida, Orlando, FL 32816 \\ ****** NanoMEGAS SPRL, Blvd Edmond Machtens 79, B-1080 Brussels, Belgium
}

Electron backscatter diffraction (EBSD) technique in a scanning electron microscope (SEM) is often employed for the structural characterization of thin polycrystalline films and texture acquisition of surfaces for metals and minerals. However, the EBSD-SEM technique has limited spatial resolution (>30 nm for SEM with a Field Emission Gun) and is very sensitive to sample preparation-induced surface roughness and damage.

To overcome the above limitations, an automated technique for phase mapping and crystal orientation of polycrystalline materials has been developed recently in the TEM [1]. This technique is based on template matching of experimental electron diffraction (ED) spot patterns, wherein the acquired ED patterns from several sample locations are compared via cross-correlation matching models with pre-calculated simulated templates to reveal local crystal orientation(s) and phase(s). The dedicated system, called ASTAR, allows crystal orientation and phase identification of regions of up to $10 \mu \mathrm{m}^{2}$, with a step size/resolution ranging from 1-20 nm depending on the gun type (FEG or $\mathrm{LaB}_{6}$ ). So far, very promising results have been obtained for phase and orientation maps of pure metals, alloys and semiconductors [1-2]. In order to improve the quality of the acquired diffraction patterns and the resulting high resolution orientation-phase maps, particularly for complex structures, beam precession is used. The precession of the beam has also been shown to reduce the occurrence of $180^{\circ}$ ambiguities related to highly symmetric spot patterns from the zero order Laue zone. This is because reflections from higher order Laue zones may be excited in PED patterns [2].

In our study we have used the ASTAR system to analyze the texture of $\mathrm{Pt}, \mathrm{Cu}$, and $\mathrm{W}$ thin films prepared by sputter deposition onto oxidized $\mathrm{Si}$ substrates. The $\mathrm{Cu}$ and $\mathrm{W}$ films were encapsulated with an under-and an overlayer of $20 \mathrm{~nm}$ sputtered $\mathrm{SiO}_{2}$. The work was performed in a JEOL 2010F TEM equipped with the ASTAR system in nanobeam mode ( $1 \mathrm{~nm}$ probe size). It is of interest to note that not only the texture may be inferred from the maps (Fig.1-3) but also the grain size is clearly revealed with such measurements. On the other hand, from conventional bright field images grain boundaries are difficult to distinguish because of pronounced dynamic diffraction contrast effects. Accurate crystallography studies can be performed in such high resolution orientation maps, including complete crystallographic characterization of boundary types (e.g., twins in Fig. 3).

References

[1] E.F. Rauch et al., Microsc. Microscopy and Analysis, Issue 93, November (2008), S5.

[2] E.F. Rauch et al., Special Issue on "Precession Electron Crystallography" of Zeits. Krist. 225, (2010) issue 2-3. 
(a)

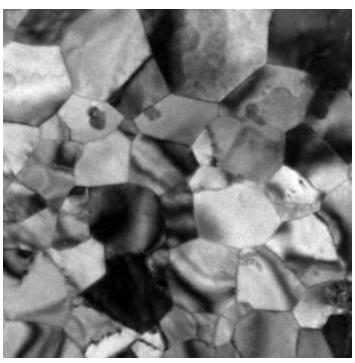

(b)

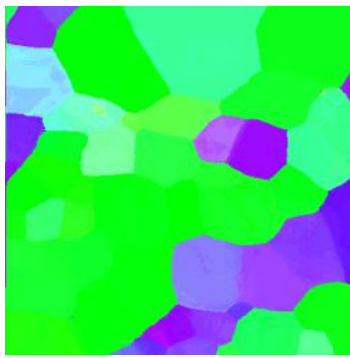

(c)

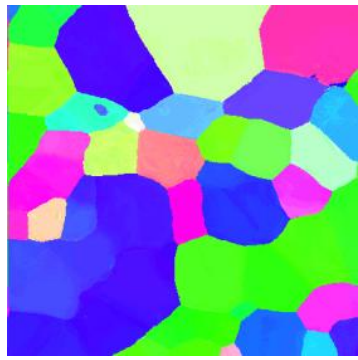

(d)

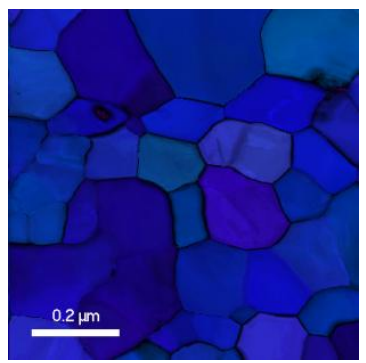

FIG.1 (a) Bright field TEM image of a $50 \mathrm{~nm}$-thick Pt film sputter deposited on oxidized Si, the corresponding orientation maps (b-d). Colors refer to inverse pole figure for the $\mathrm{x}(\mathrm{b}), \mathrm{y}(\mathrm{c})$ and $\mathrm{z}(\mathrm{d})$ axes, respectively. A [111] fiber texture is clearly seen in (d).

(a)

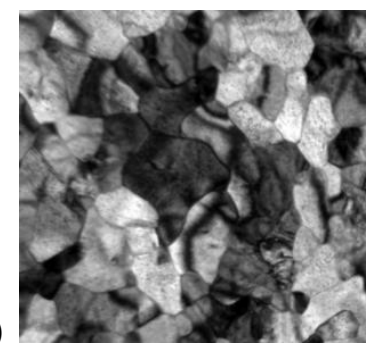

(b)

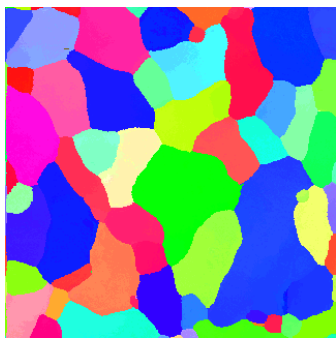

(c)

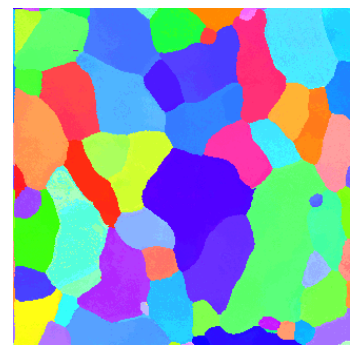

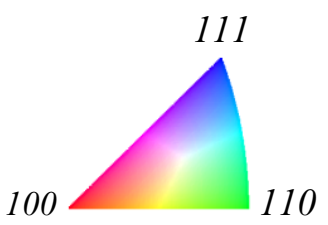

FIG. 2. (a) Bright field TEM image of a 40 nm-thick W Film sputter deposited on oxidized Si and encapsulated with $20 \mathrm{~nm}$ of sputtered $\mathrm{SiO}_{2}$, (b-c) the corresponding orientations maps (b-c).Colors refer to inverse pole figures for the $\mathrm{z}(\mathrm{b})$ and $\mathrm{x}(\mathrm{c})$ axes, respectively.

(a)

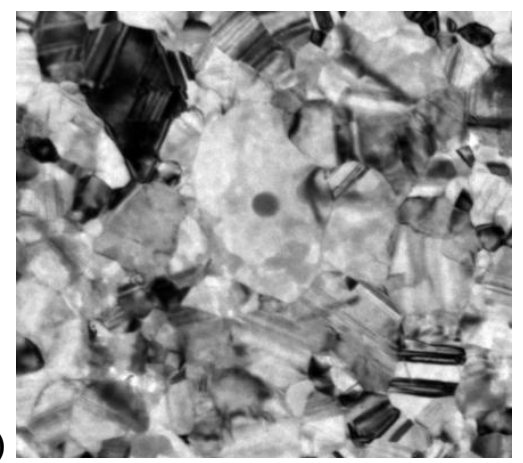

(b)

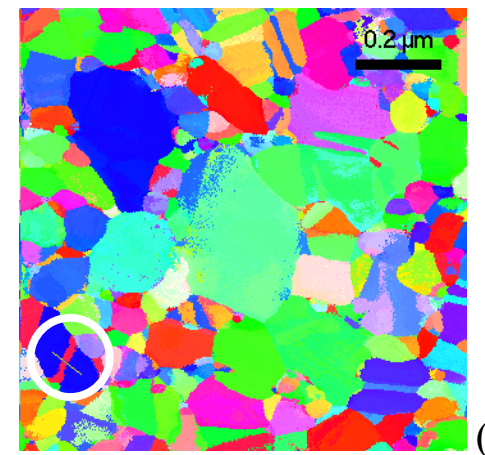

(c)

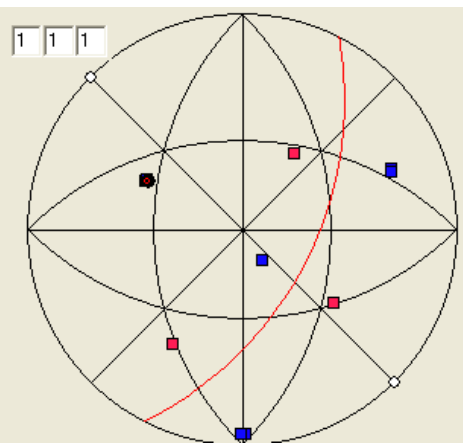

FIG. 3 Bright field TEM image of a 50 nm-thick $\mathrm{Cu}$ film encapsulated with $20 \mathrm{~nm}$ of sputtered $\mathrm{SiO}_{2}$ and (b) corresponding orientation map along y. The area circled in white in the lower left contains a twin characterized by a $60^{\circ}$ misorientation around the [111] common pole (c). Misorientation (d) is calculated along the thin line that crosses the twin boundary

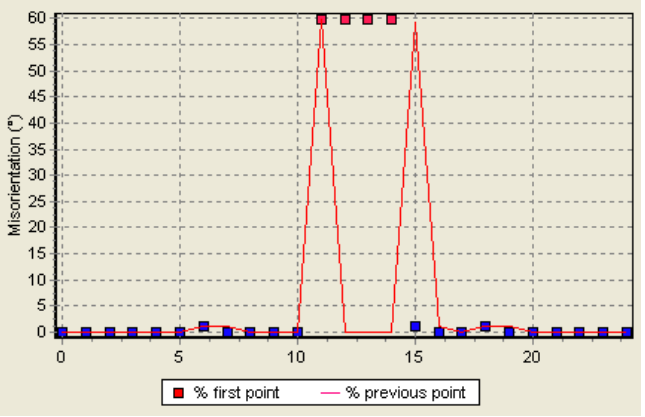

